\title{
aㅔ
}

CLINICAL REPORT

\section{Multidisciplinary approach to oral rehabilitation with dental implants after gunshot injury: A clinical report}

\author{
Lélis Gustavo Nícoli, MSc, ${ }^{a}$ Suzane Cristina Pigossi, MSc, ${ }^{b}$ Raphael Ferreira de Souza Bezerra Araújo, MSc, \\ Cláudio Marcantonio, $\mathrm{PhD}^{d}$ Élcio Marcantonio, $\mathrm{PhD}^{\mathrm{e}}$ and Élcio Marcantonio, $\mathrm{Jr} \mathrm{PhD}^{\mathrm{f}}$
}

Gunshot injuries can have devastating consequences, including extensive bone and soft tissue destruction in the oral and maxillofacial region, ${ }^{1}$ creating difficulties for rehabilitation with endosseous dental implants. ${ }^{2}$ Dental implants have been used to retain and support fixed complete dental prostheses, improving the retention and stability of the prosthetic rehabilitation. Implant-supported restorations bring functional and psychological advantages for the patients, enhancing their comfort and self-confidence. ${ }^{3}$ Delayed, early, and immediate loading of implant-fixed complete dental prostheses have been described. ${ }^{4,5}$ In immediate loading, the prosthesis is placed as soon as the implant is inserted, which has some advantages, including more rapid prosthetic replacement and reduced morbidity, treatment time, and number of surgical procedures. ${ }^{6-8}$ A review of 15 randomized controlled trials found no clinically important differences in prosthesis and implant failure or bone loss among the loading protocol types. ${ }^{9}$

Bone height in the maxilla is usually limited by the extended nasal cavity and maxillary sinus pneumatization, which hinders the ideal 3-dimensional position of the implants. ${ }^{10}$ Several techniques have been used to increase bone volume in the maxilla, including nasal floor elevation and maxillary sinus augmentation. ${ }^{11,12}$ In these procedures, the nasal mucosa and/or the maxillary sinus membrane are carefully elevated, and the cavity is filled with bone graft material. Recent studies have shown that both techniques are effective in maxillary bone reconstruction, and their implant success rates were similar. ${ }^{13-16}$

This clinical report describes a multidisciplinary approach for the treatment of a patient with edentulism and severe atrophy of both arches because of a gunshot injury by using implant-fixed complete dental prosthesis.

\section{CLINICAL REPORT}

A 63-year-old man, a heavy smoker (more than 20 cigarettes/day for 45 years), in good general health, was admitted to the School of Dentistry at Araraquara for oral rehabilitation with dental implants (Fig. 1). The patient had experienced a gunshot injury affecting his mandible

\footnotetext{
${ }^{a}$ Doctoral student, Department of Diagnosis and Surgery, São Paulo State University, Araraquara, Brazil. bDoctoral student, Department of Diagnosis and Surgery, São Paulo State University, Araraquara, Brazil. ${ }^{\mathrm{C}}$ Doctoral student, Department of Dental Materials and Prosthesis, São Paulo State University, Araraquara, Brazil.

${ }^{\mathrm{d}}$ Assistant Professor, Dental Graduate Program, University Centre of Araraquara, Araraquara, Brazil.

${ }^{\mathrm{e}}$ Assistant Professor, Dental Graduate Program, University Centre of Araraquara, Araraquara, Brazil.

${ }^{\mathrm{f}}$ Full Professor, Department of Diagnosis and Surgery, São Paulo State University, Araraquara, Brazil.
} 

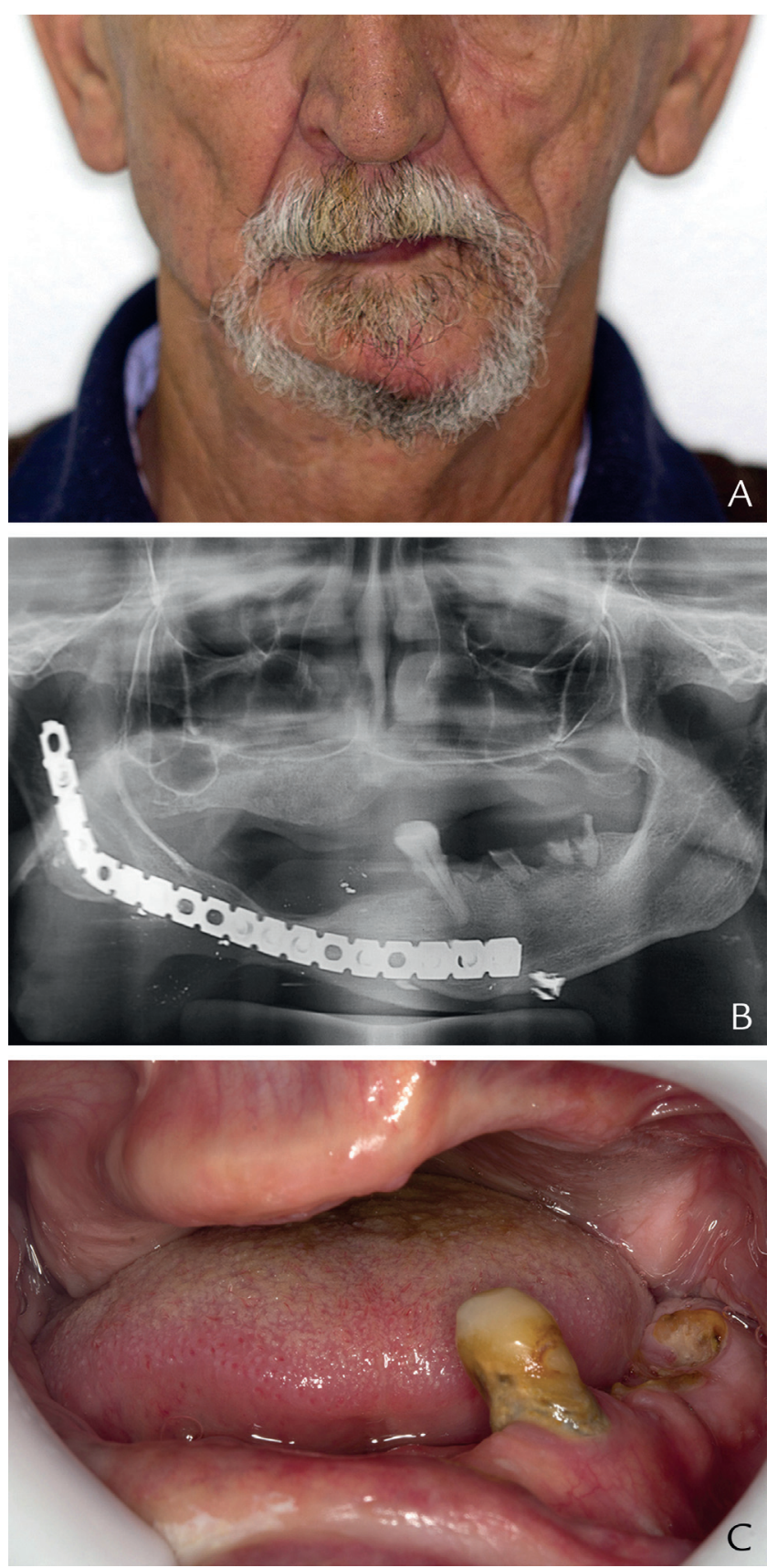

Figure 1. Pretreatment. A, Extraoral view. B, Panoramic radiograph shows extensive bone defect in mandibular right side associated with reconstruction plate and vertical bone resorption on left side of maxilla. C, Intraoral view.

23 years previously and had received a reconstruction plate for mandibular fixation at that time. After the reconstruction, the patient's oral rehabilitation involved a removable dental prosthesis in both arches. However, the mandibular atrophy impaired the performance of the prosthesis because of insufficient retention, limiting the patient's mastication and speech.
A clinical examination revealed an edentulous maxilla and some residual roots in the mandible. An extensive bone defect associated with the reconstruction plate used for the mandibular fixation after the gunshot injury was observed in the right side of the mandibular arch. In the maxilla, vertical bone resorption was extensive on the left side because of the masticatory forces exerted over more than 2 decades between the edentulous maxillary alveolar ridge and the remaining antagonist teeth (Fig. 1).

First, presurgical prosthetic planning for fabricating an implant-fixed complete dental prosthesis was executed, following conventional clinical steps. The vertical dimension was adaptively restored because of the patient's anatomic limitations, which included an altered condylar hinge position, limited mouth opening, and tissue loss on the right side of the lower lip. An immediately loaded implant-fixed complete dental prosthesis in the mandible associated with a complete denture prosthesis in the maxilla was proposed to restore the occlusal vertical dimension before providing definitive maxillary and mandibular implant-supported prostheses. The mandibular rehabilitation at this stage was limited to the right mandibular second premolar only to avoid excessive masticatory force on the atrophic side of the mandible. The right mandibular second premolar was maintained in infraocclusion, and the masticatory forces were distributed from the right mandibular first premolar to the left mandibular first molar. The reconstruction plate was not removed before placement of the dental implants. This was to avoid the surgery necessary for this procedure, as implants could not be installed in this region because of limited bone availability. After the clinical evaluation, a transparent autopolymerizing acrylic resin multifunctional guide was made by duplicating the mandibular wax base and tooth arrangement.

The height, diameter, and distribution of the dental implants were planned from cone beam computerized tomography (CBCT) images. Local anesthesia was induced using a $4 \%$ articaine solution with epinephrine (1:100 000 dilution; articaine 100; Nova DFL). An intraoral crestal incision was initiated in the left molar area and extended to the second premolar area on the opposite side. Subperiosteal dissection was performed to expose the mental foramina, and the remaining teeth were extracted. Prior to implant site preparation, the residual ridge crest of the mandible was flattened with a rotating bur under sterile saline solution irrigation to ensure sufficient space to accommodate the prosthetic requirements. The multifunctional guide was used to delimit the area to be implanted. The drilling sequence followed the manufacturer's recommendation, and 5 external hexagon $3.75 \times 1.5 \mathrm{~mm}$ implants (Conexão 


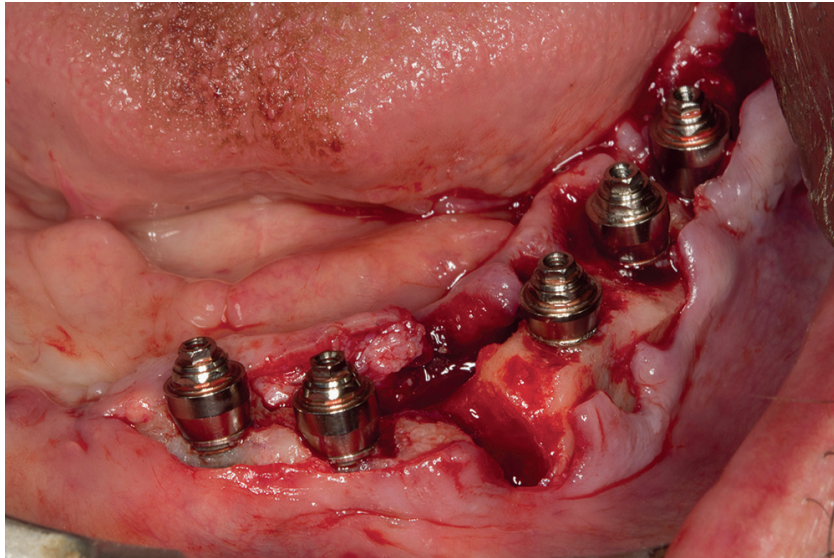

Figure 2. Five dental implants with abutments installed in mandible (left side) to avoid excessive masticatory force on atrophic side.

Sistema de Próteses) were placed. The insertion torque for all implants was approximately $45 \mathrm{Ncm}$, which allowed the immediate loading approach. Sutures were made using 4-0 silk (4-0 silk; Ethicon). The abutments (Micro Unit; Conexão Sistema de Próteses) were installed (Fig. 2), and dental implant impressions were made using the multifunctional guide. The metal framework fabrication was made by waxing and conventional casting of $\mathrm{Ni}-\mathrm{Cr}$ alloy.

On the day after the surgery, the metal framework and teeth were evaluated, and the prosthetic adaptation and esthetic/functional parameters were found to be adequate. After 48 hours, the mandibular implant-fixed complete dental prosthesis and the maxillary removable complete denture prosthesis were delivered, and occlusal adjustment was made (Fig. 3). The patient was scheduled for a follow-up visit every 4 months for removal, evaluation, and cleaning of the prosthesis and examination of the implants.

Two months after the mandibular surgery, a CBCT of the posterior maxilla revealed pneumatization of the left maxillary sinus and severe crestal resorption on the left side of the maxilla, resulting in close proximity between the alveolar crest and nasal cavity (Fig. 4). A maxillary inlay bone graft using the nasal floor and maxillary sinus augmentation technique was thus proposed to allow dental implant placement in this region.

The leukocytic-platelet rich fibrin (L-PRF) was prepared as described by Choukroun et $\mathrm{al}^{17}$ in fragment or membrane forms before the surgical procedure. Under infiltrative local anesthesia, a full-thickness mucoperiosteal flap was raised to gain access to the lateral wall of the sinus. The lateral wall cortical bone was removed using diamond round burs with sterile saline irrigation. The sinus membrane was elevated with special curettes (Cureta para Levantamento de Seio; Neodent) and the medial maxillary sinus wall was removed, allowing nasal cavity access. Nasal mucosal elevation was then performed using the same curettes. The elevation extension was determined by the position of the most mesial/ distal dental implant. The L-PRF membrane was placed over both sinus and nasal membranes to prevent perforation by the particulate graft (Fig. 5). The sinus and nasal cavities were filled with deproteinized bovine bone material (DBBM) and L-PRF (0.25- to 1-mm particle size) using a 2:1 ratio. ${ }^{18,19}$ The L-PRF membrane also covered the access window before flap closure to improve soft tissue healing. Flap closure was completed using 4.0-nylon interrupted sutures (ETHILON Suture; Ethicon). Standard sinus lifting postoperative instructions were prescribed. The sutures were removed 10 days after the surgery. A healing time of 8 months was indicated to ensure adequate bone formation.

However, before the patient's healing time was complete, in situ oral squamous cell carcinoma was diagnosed in the patient, localized to the left retromolar area of the mandible. Because of the extensive oncological treatment, the dental treatment had to be
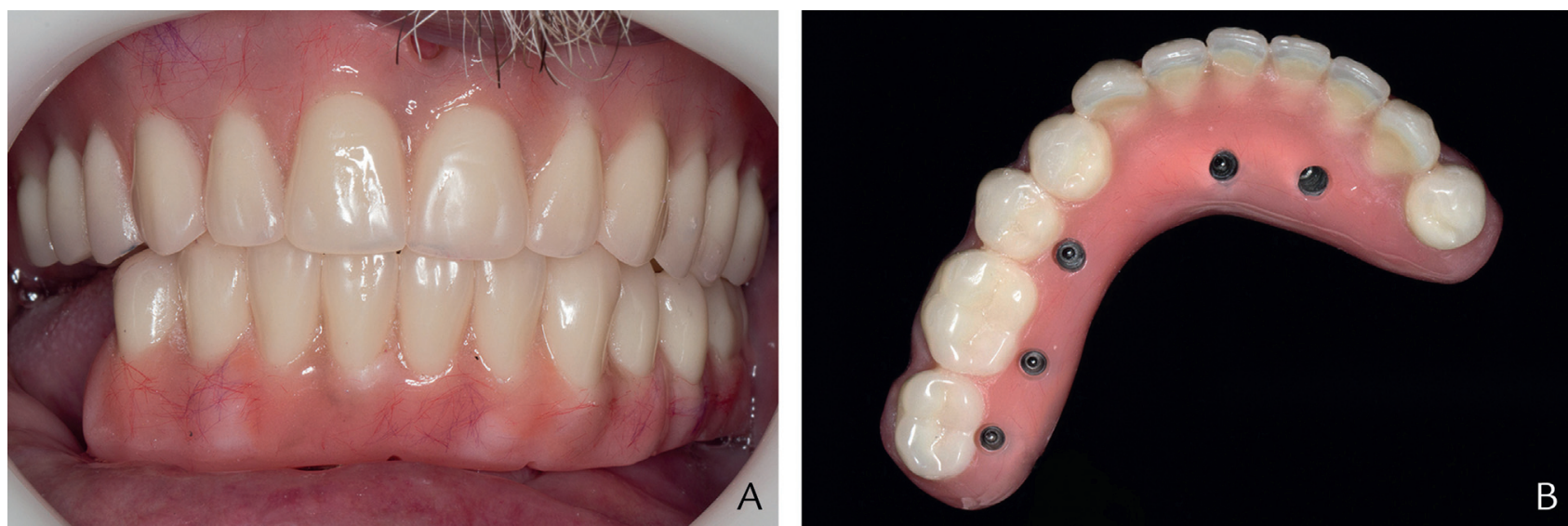

Figure 3. A, Mandibular implant-fixed complete dental prosthesis and maxillary denture installed in first treatment phase. B, Mandibular implant-fixed complete dental prosthesis modification design. 


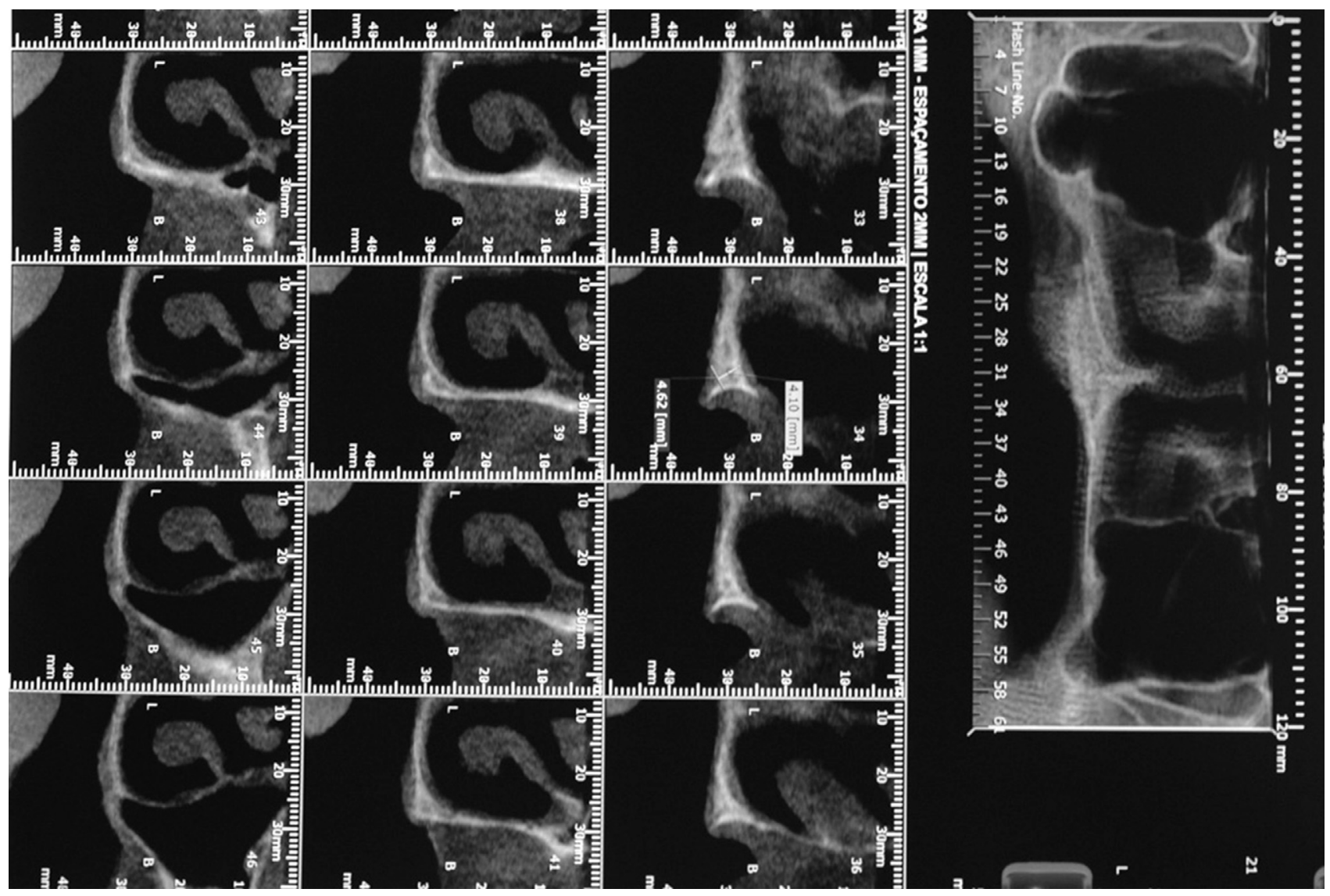

Figure 4. Maxillary cone beam computerized tomography images. Pneumatization of left maxillary sinus associated with severe crestal resorption on maxilla left side.

interrupted. For the cancer treatment, the lesion was completely excised and chemotherapy without bisphosphonates was prescribed. Two years after the cancer diagnosis, following the same approach described in the first surgical phase, a total of 6 morse cone connection implants (Conexão Sistemas de Prótese) were placed, $3(3.75 \times 11.5 \mathrm{~mm})$ on the left and $3(3.5 \times 10 \mathrm{~mm})$ on the right side of the maxilla.

Definitive impressions and abutment selections were made 6 months after implant healing. A maxillary metal framework was fabricated, and the tooth arrangements for maxillary and mandibular definitive fixed implant protocol prosthesis were fabricated to maintain the occlusal vertical dimension established and confirmed with the interim prosthesis. At the last follow-up visit (34 months from the implant-fixed prosthesis installation in the mandible and 4 months from that in the maxilla), the patient reported improvement in his masticatory function, esthetics, and quality of life and expressed satisfaction with the definitive result of the treatment. No prosthesis failure or radiograph signs of peri-implant disease were found in this follow-up visit (Figs. 6, 7)

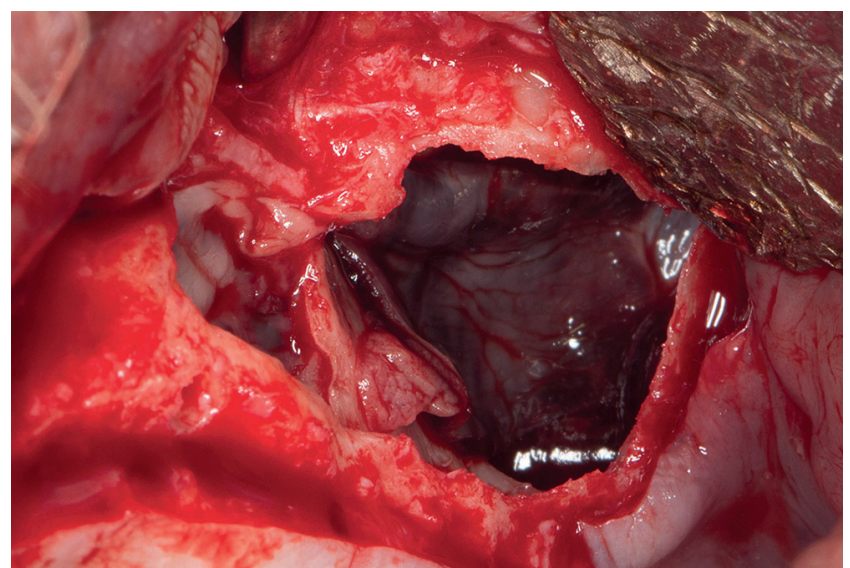

Figure 5. Internal view of the maxillary sinus and nasal cavity coated with leukocytic-platelet rich fibrin (L-PRF) membrane to prevent perforation by particulate graft.

\section{SUMMARY}

After 23 years of edentulism, the patient showed alteration in the intermaxillary relationship associated with bone/soft tissue atrophy in both arches. An immediate 


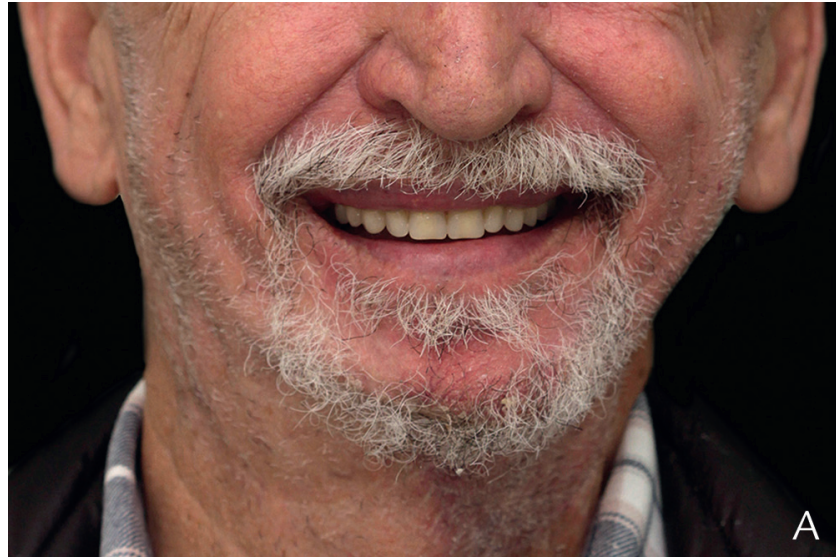

Figure 6. Post-treatment images. A, Extraoral view. B, Intraoral view.

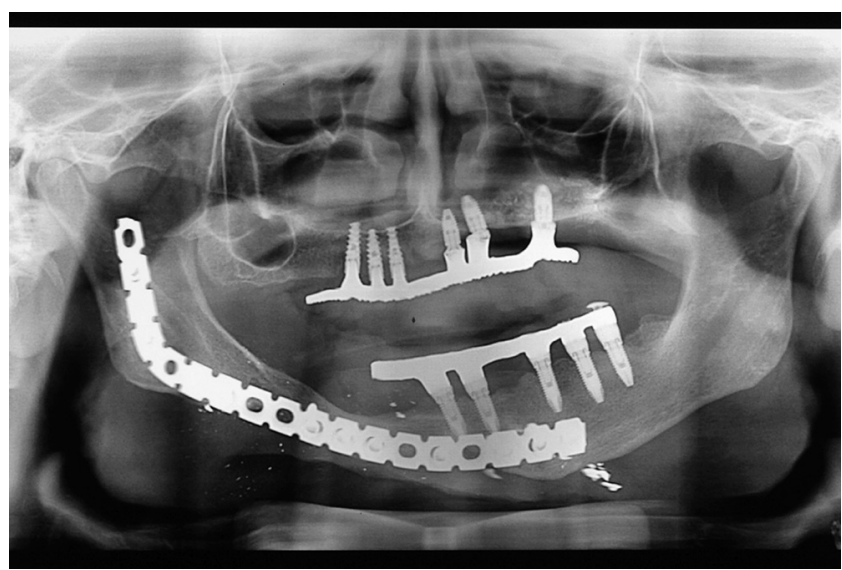

Figure 7. Panoramic radiograph after 2 years and 10 months in mandible and 4 months in maxilla from implant-fixed prosthesis installation.

loading interim implant-fixed complete dental prosthesis in the mandible associated with a maxillary removable complete denture prosthesis was initially provided to restore the intermaxillary relation. Nasal floor elevation and maxillary sinus augmentation were subsequently performed. Definitive implant-fixed complete dental prostheses were placed in both arches.

\section{REFERENCES}

1. Motamedi MH. Primary management of maxillofacial hard and soft tissue gunshot and shrapnel injuries. J Oral Maxillofac Surg 2003;61:1390-8.

2. Oliveira LB, Gabrielli MA, Gabrielli MF, Pereira-Filho VA. Implant-supported rehabilitation after treatment of atrophic mandibular fractures: report of two cases. Oral Maxillofac Surg 2015;19:427-31.

3. Lopez CS, Saka CH, Rada G, Valenzuela DD. Impact of fixed implant supported prostheses in edentulous patients: protocol for a systematic review. BMT Open 2016:6:1-4.

4. Turkyilmaz I, Tozum TF, Tumer C. Early versus delayed loading of mandibular implant-supported overdentures: 5-year results. Clin Implant Dent Relat Res 2010;12:39-46.

5. Xu L, Wang X, Zhang Q, Yang W, Zhu W, Zhao K. Immediate versus early loading of flapless placed dental implants: a systematic review. J Prosthet Dent 2014;112:760-9.

6. Koirala DP, Singh SV, Chand P, Siddharth R, Jurel SK, Aggarwal H, et al. Early loading of delayed versus immediately placed implants in the anterior

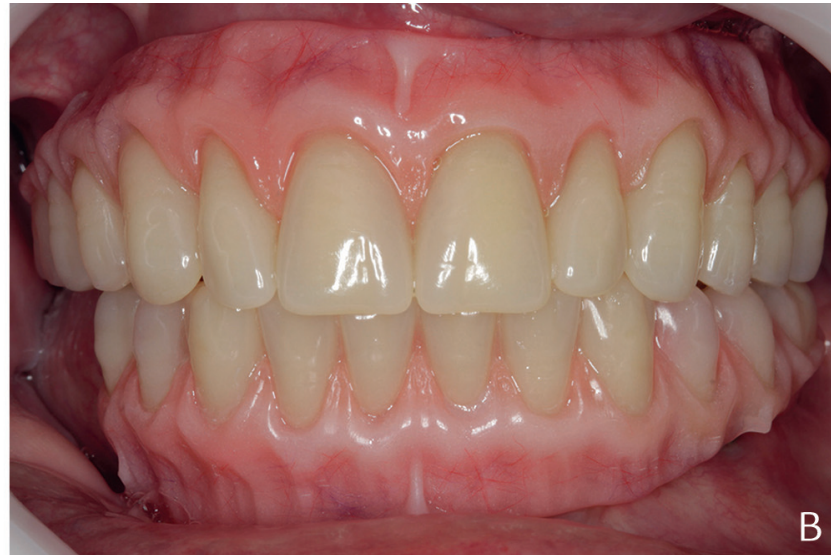

mandible: a pilot comparative clinical study. J Prosthet Dent 2016;116: 340-5.

7. Papaspyridakos P, Chen CJ, Chuang SK, Weber HP. Implant loading protocols for edentulous patients with fixed prostheses: a systematic review and meta-analysis. Int J Oral Maxillofac Implants 2014:29:256-70.

8. Faria PE, Masalskas B, Heyden A, Rasmusson L, Salata LA. Immediate loading of implants in the edentulous mandible: a multicentre study. Oral Maxillofac Surg 2016;20:385-90.

9. Esposito M, Grusovin MG, Maghaireh H, Worthington HV. Interventions for replacing missing teeth: different times for loading dental implants. Cochrane Database Syst Rev 2013;3:1-94.

10. Misch CE. Density of bone: effect on treatment plans, surgical approach, healing, and progressive bone loading. Int J Oral Implantol 1990;6:23-31.

11. Mardinger O, Chaushu G, Sigalov S, Herzberg R, Shlomi B, Schwartz Arad D. Factors affecting changes in sinus graft height between and above the placed implants. Oral Surg Oral Med Oral Pathol Oral Radiol Endod 2011;111:6-11.

12. Aghaloo TL, Moy PK. Which hard tissue augmentation techniques are the most successful in furnishing bony support for implant placement? Int J Oral Maxillofac Implant 2007;22:49-70.

13. El-Ghareeb M, Pi-Anfruns J, Khosousi M, Aghaloo T, Moy P. Nasal floor augmentation for the reconstruction of the atrophic maxilla: a case series. J Oral Maxillofac Surg 2012;70:235-41.

14. Garcia-Denche JT, Abbushi A, Hernandez G, Fernandez-Tresguerres I, Lopez-Cabarcos E, Tamimi F. Nasal floor elevation for implant treatment in the atrophic premaxilla: a within-patient comparative study. Clin Implant Dent Relat Res 2015;17:520-30.

15. Lorean A, Mazor Z, Barbu H, Mijiritsky E, Levin L. Nasal floor elevation combined with dental implant placement: a long-term report of up to 86 months. Int J Oral Maxillofac Implants 2014;29:705-8.

16. Mazor Z, Lorean A, Mijiritsky E, Levin L. Nasal floor elevation combined with dental implant placement. Clin Implant Dent Relat Res 2012;14: 768-71.

17. Choukroun J, Diss A, Simonpieri A, Girard MO, Schoeffler C, Dohan SL, et al. Platelet-rich fibrin (PRF): a second-generation platelet concentrate. part V: histologic evaluations of PRF effects on bone allograft maturation in sinus lift. Surg Oral Med Oral Pathol Oral Radiol Endod 2006;101:299-303.

18. Zhang Y, Tangl S, Huber CD, Lin Y, Oiu L, Rausch-Fan X. Effects of Choukroun's platelet-rich fibrin on bone regeneration in combination with deproteinized bovine bone mineral in maxillary sinus augmentation: a histological and histomorphometric study. J Craniomaxillofac Surg 2012;40: 321-8.

19. Bolukbasi N, Ersanli S, Keklikoglu N, Basegmez C, Ozdemir T. Sinus augmentation with platelet-rich fibrin in combination with bovine bone graft versus bovine bone graft in combination with collagen membrane. J Oral Implantol 2015;41:586-95.

Corresponding author:

Dr Elcio Marcantonio Junior

Department of Diagnosis and Surgery

São Paulo State University (UNESP)

Humaitá, 1680

Araraquara, SP -14801-903

BRAZIL

Email: elciojr@foar.unesp.br

Copyright (C) 2017 by the Editorial Council for The Journal of Prosthetic Dentistry. 